Title: Inverse Spectral Problems. 1-D, Theoretical Results

Name: $\quad$ Mourad Sini

Affil./Addr.: $\quad$ RICAM, Austrian Academy of Sciences,

69 Altenbergerstrasse, A 4040, Linz, Austria.

Email: mourad.sini@oeaw.ac.at

\title{
Inverse Spectral Problems. 1-D, Theoretical Results
}

\section{Introduction}

Let $p, q$ and $\rho$ be real valued, bounded and measurable functions defined on the interval $(0,1)$ with $p$ and $\rho$ positive. We denote by $\lambda_{i}$ and $\phi_{i}, i \in \mathbb{N}$, the associated eigenvalues and the $L_{\rho}^{2}(0,1)$-orthonormal eigenfunctions of the Sturm-Liouville problem:

$$
\left\{\begin{array}{l}
-\left(p u^{\prime}\right)^{\prime}+q u-\lambda \rho u=0, \text { in }(0,1), \\
u(0)=u(1)=0
\end{array}\right.
$$

where $L_{\rho}^{2}(0,1)$ is the $L^{2}(0,1)$-space with the scalar product $(f, g):=\int_{0}^{1} f(x) g(x) \rho(x) d x$. If we replace in $(1), u(1)=0$ by $\left(p u^{\prime}\right)(1)=0$, then we have another sequence of eigenvalues and eigenfunctions, which we denote by $\left(\mu_{i}\right)_{i=1}^{\infty}$ and $\left(e_{i}\right)_{i=1}^{\infty}$ respectively. In the next sections, we will discuss the following two types of inverse spectral problems

1. The Borg-Levinson inverse spectral problem. It consists of the reconstruction of some of the three coefficients $p, q$ and $\rho$ from the spectral data $\left(\lambda_{i}, \mu_{j}\right)_{i=1}^{\infty}$.

2. The Gelfand inverse spectral problem. It consists of the reconstruction of some of the three coefficients $p, q$ and $\rho$ from the spectral data $\left(\lambda_{i},\left|\left(p \phi_{i}^{\prime}\right)(0)\right|\right)_{i=1}^{\infty}$.

Different boundary conditions rather than the one in (1) can be taken. In addition, other types of inverse spectral problems have been also considered in the literature. 
We can cite among others, for the case $p=\rho=1$ for instance, the one related to the spectral data $\left(\lambda_{n}, \log \frac{\left|\phi_{n}^{\prime}(1)\right|}{\left|\phi_{n}^{\prime}(0)\right|}\right)_{n \in \mathbb{N}}$ or to the mixed data, i.e. given $\left(\lambda_{n}\right)_{n \in \mathbb{N}}$ and the a priori information that $q$ is symmetric with respect the middle point $x_{0}:=\frac{1}{2}$. We cite also the spectral data consisting of the sequence $\left(\lambda_{n}\right)_{n \in \mathbb{N}}$ and the nodal points (or the zeros of the corresponding eigenfunctions $\phi_{n}$ ), called the inverse nodal problem. More information on these cases can be found in the following references [3, 8, 9, 10] for instance. In this paper, we focus only on the Gelfand and the Borg-Levinson spectral problems. An observation we can make is that we cannot obtain more than one of the three coefficients $p, q$ and $\rho$. To see this, assume in addition that $p$ and $\rho$ are of class $C^{2}(0,1)$. We define the Liouville transformation $y(x):=\frac{1}{L} \int_{0}^{x} \sqrt{\frac{\rho}{p}}(t) d t, x \in[0,1]$, with $L:=\int_{0}^{1} \sqrt{\frac{\rho}{p}}(t) d t$. Using this transformation as a coordinate transformation, we can verify that the Gelfand as well as the Borg-Levinson spectral data related to the general form Sturm Liouville equation $-\left(p u^{\prime}\right)^{\prime}+q u-\lambda \rho u=0$, in $(0,1)$, are equal to the ones of the normal form Sturm Liouville equation $-u^{\prime \prime}+V u-\lambda u=0$, in $(0,1)$, where $V$, which is a bounded and measurable real valued function, is given as a combination of the three coefficients $p, q$ and $\rho$. Note that this transformation is not valid for discontinuous coefficients $p$ and $\rho$.

The literature on these $1 d$ inverse spectral problems is huge. So, instead of reviewing the known results, we chose to review some of the popular ideas for solving the Gelfand and the Borg-Levinson problem considering the Sturm Liouville equation of the normal form ${ }^{1}$. Indeed, in section 2 , we mention the asymptotic expansion technique used for the first time by Borg and then simplified by Levinson at the end of the 40s, see $[2,7]$. In section 3 , we explain briefly the integral equation method by Gelfand and Levitan introduced in the 50s for solving the Gelfand inverse spectral problems,

\footnotetext{
${ }^{1}$ We assumed the potential $V$ to be bounded but, of course, this is not optimal and many of the results stated here are known for potentials belonging to larger spaces.
} 
see [4]. During the period from the 50s till the 80s these two approaches have been extensively studied by many authors, see the references $[8,9,10]$ for more information on these methods and the related results till mid 80s. In section 4 , we consider the method of the C-property by Ramm, see [11], and in section 5, the so called boundary control method by Belichev and Kurylev both introduced in the mid 80s, see [1] for the original version and [5] for a different presentation. We describe these methods for proving the uniqueness results. However, we warm the reader that two of them (the Gelfand-Levitan and the boundary control methods) are reconstructive. In addition, it is worth mentioning that the boundary control method has been also stated for the multidimensional problems, see [1]. Our goal in this paper is to explain the ideas by highlighting, with details, the link between the spectral data and the main mathematical tool proposed in each of the mentioned approaches. Regarding the step from the main mathematical tool to the final result, either we give some details, when it is possible, or we provide an appropriate reference.

The starting point for solving these problems is the following asymptotic formulas for the eigenmodes $\left(\lambda_{n}, \phi_{n}\right)$ in terms of $n$, see [2] for the original proof or [6] for a more simplified proof using Volterra type integral equations in addition to some complex analysis techniques.

Lemma 1. The sequence of eigenvalues $\left(\lambda_{n}\right)_{n \in \mathbb{N}}$ has the following asymptotic expression:

$$
\lambda_{n}=n^{2} \pi^{2}+\int_{0}^{1} V(t) d t+O\left(\frac{1}{n}\right)
$$

and the sequence of normalized eigenfunctions $\left(\phi_{n}(x)\right)_{n \in \mathbb{N}}$ behaves as follows

$$
\phi_{n}(x)=\sqrt{2} \sin (n \pi x)+O\left(\frac{1}{n}\right) \text { and } \phi_{n}^{\prime}(x)=\sqrt{2} n \pi \cos (n \pi x)+O(1)
$$

for $n \rightarrow \infty$, uniformly for $x \in[0,1]$. 


\section{The asymptotic expansion technique}

The original idea of Borg and as simplified by Levinson for solving the Borg-Levinson inverse spectral problem goes as follows. We introduce the Cauchy problem satisfied by $u:=u(x, \lambda)$

and the one satisfied by $v:=v(x, \lambda)$

$$
\left\{\begin{array}{l}
-u^{\prime \prime}+V u-\lambda u=0, \quad \text { in }(0,1), \\
u(0, \lambda)=0, \text { and } u^{\prime}(0, \lambda)=1
\end{array}\right.
$$

$$
\left\{\begin{array}{l}
-v^{\prime \prime}+V v-\lambda v=0, \quad \text { in }(0,1), \\
v(1, \lambda)=0, \text { and } v^{\prime}(1, \lambda)=1
\end{array}\right.
$$

Similar to the asymptotic expansion (3), we have

$$
\left\{\begin{array}{l}
u(x, \lambda)=\frac{\sin (\sqrt{\Re \lambda} x)}{\sqrt{\Re \lambda}}+O\left(\frac{e^{|\Im \lambda| x}}{|\Re \lambda|^{2}}\right) \\
u^{\prime}(x, \lambda)=\sin (\sqrt{\Re \lambda x})+O\left(\frac{e^{|\Im \lambda| x}}{|\Re \lambda|}\right)
\end{array}\right.
$$

for $|\lambda| \rightarrow \infty$, uniformly in $[0,1]$. Note that $\frac{\phi_{n}}{\phi_{n}^{\prime}(0)}$ satisfies (4) and $\frac{\phi_{n}}{e_{n}^{\prime}(1)}$ satisfies $(5)$ with $\lambda:=\lambda_{n}$. We define the characteristic function $w(\lambda):=u(1, \lambda)$. It is an entire function and has as zeros the eigenvalues $\lambda_{n}, n=1,2, \ldots$ The expansion (6) implies that $w(\lambda)$ is entire of order $1 / 2$ and hence, by the Hadamard's factorization theorem it is completely characterized by its zeros, $\lambda_{n}, n=1,2, \ldots$, i.e. $w(\lambda)=C(V) \Pi_{n=1}^{\infty}\left(1-\frac{\lambda}{\lambda_{n}}\right)$ with some constant $C(V)$. Let now $V_{1}$ and $V_{2}$ have the same Borg-Levinson spectral data. We define $u_{j}(x, \lambda)$ as the solution of (4) for potential $V_{j}$. Hence the corresponding characteristic functions $w_{j}(\lambda)$, satisfy $w_{1}=w_{2}=: w$ as functions since $C\left(V_{1}\right)=C\left(V_{2}\right)$ from the first property in (6). We define also $v_{j}(x, \lambda)$ to be the solution of $(5)$ for the potential $V_{j}$. From (4) and (5), we deduce that

$$
u_{j}\left(x, \lambda_{n}\right)=C_{n} v_{j}\left(x, \lambda_{n}\right)
$$

where $C_{n}$ is independent of $j, j=1,2$. Indeed, it is clear that $u_{j}\left(x, \lambda_{n}\right)=C_{n}^{j} v_{j}\left(x, \lambda_{n}\right)$, (since $u_{j}\left(x, \lambda_{n}\right)=\frac{\phi_{n}^{j}(x)}{\left(\phi_{n}^{j}\right)^{\prime}(0)}$ and $\left.v_{j}\left(x, \lambda_{n}\right)=\frac{\phi_{n}^{j}(x)}{\left(\phi_{n}^{j}\right)^{\prime}(1)}\right)$. But $u_{j}^{\prime}(1, \lambda)$ is the characteristic 
function associated to the mixed boundary conditions $u(0)=u^{\prime}(1)=0$. Hence, similar to $w(\lambda)$, it is characterized by its eigenvalues $\mu_{n}, n=1,2, \ldots$ Since $u_{j}^{\prime}\left(1, \lambda_{n}\right)=C_{n}^{j}$ then $C_{n}^{1}=C_{n}^{2}=: C_{n}$, for $n$ in $\mathbb{N}$. Let us mention that, for this approach, the equality of the Borg-Levinson spectral data is used only to prove $(7)$ and $u_{1}(1, \lambda)=u_{2}(1, \lambda)=: w(\lambda)$.

The main new argument of Levinson, see [7], starts from here. He defines the following function

$$
H(x, \lambda):=\frac{1}{w(\lambda)} v_{2}(x, \lambda) \int_{0}^{x} u_{1}(\xi, \lambda) f(\xi) d \xi, \forall \lambda \neq \lambda_{n}
$$

where $f \in C_{0}^{1}[0,1]$. Using the property (6) and an appropriate contour of integration, he shows that

$$
\int_{\Gamma_{N}} H(x, \lambda) d \lambda-\pi i f(x) \rightarrow 0, N \rightarrow \infty
$$

where $\Gamma_{N}$ is a circle of center $\lambda=0$ and radius between $\lambda_{N}^{1 / 2}$ and $\lambda_{N}^{3 / 2}$. Applying the residue theorem to the left hand side of (9) and using the identity (7), for $j=2$, we obtain $f(x)=2 \sum_{n=1}^{\infty} \frac{u_{2}\left(x, \lambda_{n}\right) \int_{0}^{x} u_{1}\left(t, \lambda_{n}\right) f(t) d t}{C_{n} w^{\prime}\left(\lambda_{n}\right)}$. Applying the same calculations to $\frac{1}{w(\lambda)} u_{2}(x, \lambda) \int_{x}^{1} v_{1}(\xi, \lambda) f(\xi) d \xi$ instead of $H(x, \lambda)$, we obtain $f(x)=$ $2 \sum_{n=1}^{\infty} \frac{u_{2}\left(x, \lambda_{n}\right) \int_{x}^{1} u_{1}\left(t, \lambda_{n}\right) f(t) d t}{C_{n} w^{\prime}\left(\lambda_{n}\right)}$. Summing up these last two identities, we get the first expansion of $f: f(x)=\sum_{n=1}^{\infty} \frac{u_{2}\left(x, \lambda_{n}\right) \int_{0}^{1} u_{1}\left(t, \lambda_{n}\right) f(t) d t}{C_{n} w^{\prime}\left(\lambda_{n}\right)}$. Now exchanging the roles of $u_{1}$ and $v_{1}$ by $u_{2}$ and $v_{2}$, we obtain the second expansion of $f: f(x)=\sum_{n=1}^{\infty} \frac{u_{2}\left(x, \lambda_{n}\right) \int_{0}^{1} u_{2}\left(t, \lambda_{n}\right) f(t) d t}{C_{n} w^{\prime}\left(\lambda_{n}\right)}$. As a conclusion of these two expansions, we have

$$
\sum_{n=1}^{\infty} \frac{u_{2}\left(x, \lambda_{n}\right) \int_{0}^{1}\left[u_{2}\left(t, \lambda_{n}\right)-u_{1}\left(t, \lambda_{n}\right)\right] f(t) d t}{C_{n} w^{\prime}\left(\lambda_{n}\right)}=0 .
$$

Finally, using orthogonality properties of $u_{2}\left(x, \lambda_{n}\right), n \in \mathbb{N}$ and choosing $f(t):=$ $\sin (n \pi x)$, for instance, we deduce that $u_{1}\left(x, \lambda_{n}\right)=u_{2}\left(x, \lambda_{n}\right)$, in $[0,1]$, which implies that $V_{1}=V_{2}$. 


\section{The integral equation technique}

We give here the main idea of the approach by Gelfand and Levitan in their seminal paper [4], for solving the Gelfand inverse spectral problem. We start by stating the following key theorem which relates solutions of the Cauchy problems for the equations $-u^{\prime \prime}+V_{j} u-\lambda u=0, j=1,2$ via a Volterra integral operator of which kernel is the solution of a Goursat problem with potential $V_{1}-V_{2}$, see [6].

Theorem 1. Let $u_{j}(\cdot, \lambda) \in C^{2}[0,1]$ be the solutions of the hyperbolic problem:

$$
\left\{\begin{array}{l}
-u_{j}^{\prime \prime}+V_{j} u_{j}=\lambda u_{j}, \quad \text { in }(0,1), u_{j}(0, \lambda)=0, j=1,2 \\
u_{1}^{\prime}(0, \lambda)=u_{2}^{\prime}(0, \lambda) .
\end{array}\right.
$$

Let also $K \in C(\bar{\Delta})$ be the solution of the Goursat type problem

$$
\left\{\begin{array}{l}
\frac{\partial^{2}}{\partial x^{2}} K(x, t)-\frac{\partial^{2}}{\partial t^{2}} K(x, t)+\left(V_{1}(t)-V_{2}(x)\right) K(x, t)=0, \text { in } \Delta \\
K(x, 0)=0, \quad \text { in }[0,1] \\
K(x, x)=\frac{1}{2} \int_{0}^{x}\left(V_{1}(t)-V_{2}(t)\right) d t, \quad \text { in }[0,1]
\end{array}\right.
$$

where $\Delta:=\left\{(x, t) \in \mathbb{R}^{2}, 0<t<x<1\right\}$. Then we have

$$
u_{1}(x, \lambda)=u_{2}(x, \lambda)+\int_{0}^{x} K(x, t) u_{2}(t, \lambda) d t, \quad \text { in }[0,1], \lambda \in \mathbb{C} .
$$

Let us now explain how this theorem can be used to prove the uniqueness property. Assume that both the potentials $V_{1}$ and $V_{2}$ have the same eigenvalues $\lambda_{n}^{1}=\lambda_{n}^{2}$ and the same traces of eigenfunctions $\left(\phi_{n}^{1}\right)^{\prime}(0)= \pm\left(\phi_{n}^{2}\right)^{\prime}(0), n \in \mathbb{N}$. First, recall that $u_{j}\left(x, \lambda_{n}\right)=$ $\frac{\phi_{n}^{j}(x)}{\left(\phi_{n}^{j}\right)^{\prime}(0)}, j=1,2$, satisfies (10) hence they also satisfy $(12)$. Since $u_{j}\left(1, \lambda_{n}\right)=0$, then $\int_{0}^{1} K(1, t) \phi_{n}^{2}(t) d t=0, \forall n \in \mathbb{N}$, which implies from the denseness of the eigenfunctions $\phi_{n}^{2}, n \in \mathbb{N}$, in $L^{2}(0,1)$ that

$$
K(1, t)=0, t \in[0,1]
$$

Second, it is shown, see [13] for instance, that from the equality of the Gelfand spectral we have $\left(\phi_{n}^{1}\right)^{\prime}(1)= \pm C\left(\phi_{n}^{2}\right)^{\prime}(1)$ where $C$ is constant. By the asymptotic expansion in 
(6), we deduce that $C=1$. Using the representation (12) applied for $\lambda_{n}$ and taking the derivative and then the trace on the point $x_{0}=1$, we obtain $\int_{0}^{1} \frac{\partial}{\partial x} K(1, t) \phi_{n}^{2}(t) d t=$ $0, \forall n \in \mathbb{N}$, from which we deduce that

$$
\frac{\partial}{\partial x} K(1, t)=0, t \in[0,1]
$$

Resuming, we have shown that $K(x, t)$ satisfies the Cauchy problem in $\Delta$ given by the first equation in (11) and the initial conditions (13) and (14). From the uniqueness of the solutions of this Cauchy problem, see [6], we deduce that $K$ is identically zero. As a conclusion, we obtain from the last equation of (11) that $\int_{0}^{x}\left(V_{1}(x)-V_{2}(x)\right) d x=0$, in $[0,1]$ and hence $V_{1}=V_{2}$.

\section{The C-property}

A. Ramm introduced a method for proving the uniqueness property for one dimensional inverse spectral and inverse scattering problems, see [11] for more details. It is based on the following property which he called the C-property. Let $u_{j}(x, \lambda)$ be the solution of (4) for $V=V_{j}, j=1,2$, then we have the following property, see [11] for the proof.

Theorem 2. The set of products $\left(u_{1}(\cdot, \lambda) u_{2}(\cdot, \lambda)\right)_{\lambda>0}$ is dense in $L^{1}(0,1)$, i.e. let $h \in$ $L^{1}(0,1)$ such that $\int_{0}^{1} h(x) u_{1}(x, \lambda) u_{2}(x, \lambda) d x=0$ for every $\lambda>0$, then $h=0$.

Let us explain how this result answers the uniqueness question of the Gelfand inverse spectral problem. Multiplying the first equation of (4) corresponding to $j=1$ by $u_{2}(\cdot, \lambda)$ and conversely the one corresponding to $j=2$ by $u_{1}(\cdot, \lambda)$, integrating by parts and taking the difference, we obtain

$$
\int_{0}^{1}\left(V_{1}-V_{2}\right)(x) u_{1}(x, \lambda) u_{2}(x, \lambda) d x=u_{1}^{\prime}(1, \lambda) u_{2}(1, \lambda)-u_{2}^{\prime}(1, \lambda) u_{1}(1, \lambda), \forall \lambda \in \mathbb{C} .
$$

As a next step, we show that the equality of the Gelfand spectral data implies that 


$$
u_{1}^{\prime}(1, \lambda) u_{2}(1, \lambda)-u_{2}^{\prime}(1, \lambda) u_{1}(1, \lambda)=0, \forall \lambda \in \mathbb{C} .
$$

Hence the C-property, i.e. Theorem 2, implies that $V_{1}=V_{2}$.

In the following lines, we give a very short justification of (16). As we explained in section $2, u_{j}(1, \lambda)$ is completely characterized by its eigenvalues. Hence $u_{1}(1, \lambda)=u_{2}(1, \lambda)$. Remark that we need only the equality of the eigenvalues to obtain this equality. If in addition we have the equality of the traces of the eigenfunctions, then we have the equality of the derivatives. We state this in the following lemma.

Lemma 2. If the Gelfand spectral data are equal for $j=1,2$, then we have the identity

$$
u_{1}^{\prime}(1, \lambda)=u_{2}^{\prime}(1, \lambda), \forall \lambda \in \mathbb{C}
$$

Proof. We introduce the function $\bar{u}_{j}$ satisfying the problem:

$$
\left\{\begin{array}{l}
-\bar{u}_{j}^{\prime \prime}+V_{j} \bar{u}_{j}=0, \quad \text { in }(0,1), j=1,2 \\
\bar{u}_{j}(0, \lambda)=0, \bar{u}_{j}(1, \lambda)=u_{j}(1, \lambda) .
\end{array}\right.
$$

We set $w_{j}:=u_{j}-\bar{u}_{j}$ then it satisfies:

$$
\left\{\begin{array}{l}
-w_{j}^{\prime \prime}+V_{j} w_{j}=\lambda u_{j}, \quad \text { in }(0,1), j=1,2 \\
w_{j}(0, \lambda)=w_{j}(1, \lambda)=0
\end{array}\right.
$$

Multiplying (19) by $\phi_{n}^{j}$ and integrating by parts, we obtain:

$$
\int_{0}^{1} w_{j}(x) \phi_{n}^{j}(x) d x=-\frac{\lambda}{\left(\lambda-\lambda_{n}\right) \lambda_{n}}\left(\phi_{n}^{j}\right)^{\prime}(1) u_{j}(1, \lambda) .
$$

Using (20), we write $w_{j}=\sum_{1}^{\infty}\left[\int_{0}^{1} w_{j}(x) \phi_{n}^{j}(x) d x\right] \phi_{n}^{j}=-\sum_{1}^{\infty} \frac{\lambda\left(\phi_{n}^{j}\right)^{\prime}(1) u_{j}(1, \lambda)}{\left(\lambda-\lambda_{n}\right) \lambda_{n}} \phi_{n}^{j}$. Taking the derivative and the trace on the point $x=1$, we have $w_{j}^{\prime}(1, \lambda)=-\sum_{n=1}^{\infty} \frac{\lambda\left|\left(\phi_{n}^{j}\right)^{\prime}(1)\right|^{2} u_{j}(1, \lambda)}{\left(\lambda-\lambda_{n}\right) \lambda_{n}}$. From the equality of the Gelfand spectral data ${ }^{2}$ and the equality $u_{1}(1, \lambda)=u_{2}(1, \lambda)$, shown before, we see that $w_{1}^{\prime}(1, \lambda)=w_{2}^{\prime}(1, \lambda)$. Hence

\footnotetext{
${ }^{2}$ We explained in the previous section how the Gelfand spectral data imply that $\left|\left(\phi_{n}^{1}\right)^{\prime}(1)\right|=$ $\left|\left(\phi_{n}^{2}\right)^{\prime}(1)\right|, \forall n \in \mathbb{N}$.
} 


$$
u_{1}^{\prime}(1, \lambda)-u_{2}^{\prime}(1, \lambda)=\bar{u}_{1}^{\prime}(1, \lambda)-\bar{u}_{1}^{\prime}(1, \lambda)
$$

Now, remark that $\bar{u}_{j}(x, \lambda)=\overline{\bar{v}}_{j}(x) u_{j}(1, \lambda)$ where $\overline{\bar{v}}_{j}$ is the solution of (18) replacing $u(1, \lambda)$ by 1 . Hence $\bar{u}_{j}^{\prime}(1, \lambda)=\left(\overline{\bar{v}}_{j}\right)^{\prime}(1) u_{j}(1, \lambda)$. Recalling that $u_{1}(1, \lambda)=u_{2}(1, \lambda)$, the identity (21) becomes

$$
u_{1}^{\prime}(1, \lambda)-u_{2}^{\prime}(1, \lambda)=\left(\left(\overline{\bar{v}}_{1}\right)^{\prime}(1)-\left(\overline{\bar{v}}_{2}\right)^{\prime}(1)\right) u_{1}(1, \lambda)
$$

From the identities in (6) taken for $\lambda$ real and positive, the identity (22) can be written as

$$
O\left(\frac{1}{\lambda}\right)=\left[\left(\overline{\bar{v}}_{1}\right)^{\prime}(1)-\left(\overline{\bar{v}}_{2}\right)^{\prime}(1)\right]\left[\frac{\sin (\sqrt{\lambda})}{\sqrt{\lambda}}+O\left(\frac{1}{\lambda^{2}}\right)\right] .
$$

which implies that $\left(\overline{\bar{v}}_{1}\right)^{\prime}(1)-\left(\overline{\bar{v}}_{2}\right)^{\prime}(1)=0$ and hence $u_{1}^{\prime}(1, \lambda)-u_{2}^{\prime}(1, \lambda)=0$. This ends the proof of Lemma 2 .

\section{The boundary control method}

The boundary control method introduced by Belishev, see [1], is based on a combination of properties of the solutions of dynamical problems with the control theory of partial differential equations. Comparing it to the previous methods, it has the potential to be applied to the higher dimension inverse spectral and dynamical problems. The reader can refer to the review works [1] and [5] for more details. In this section, we show the main ideas of this theory needed to solve the $1 d$ Gelfand inverse spectral problem. For this, we state first the following hyperbolic problem related to our Sturm-Liouville model:

$$
\left\{\begin{array}{l}
\frac{\partial^{2} u}{\partial t^{2}}-\frac{\partial^{2} u}{\partial x^{2}}+V u=0, \text { in }(0, T) \times(0,1), \\
u(t, 0)=f(t), u(t, 1)=0, t \in(0, T) \\
u(0, x)=\frac{\partial u}{\partial t}(0, x)=0, x \in(0,1)
\end{array}\right.
$$

where $f \in H^{1}(0, T)$ such that $f(0)=0$ and $T$ is a positive constant. This problem is well posed. We set $u^{f}$ its solution. The justification of the boundary control method for the $1 d$ problems is based on the following arguments: 
1. Domain of influence of the waves. The support of $u^{f}(t, x)$ is given explicitly by the speed of propagation ${ }^{3}$ (in our case it equals 1 ), i.e. $\{(t, x) \in(0, T) \times(0,1), x<$ $t\}$. For $t>0$ fixed, we set $\Gamma_{t}:=\{x \in(0,1), x<t\}=(0, t)$.

2. Fourier expansion of the waves. We use the sequence $\left(\phi_{n}, \lambda_{n}\right)_{n \in \mathbb{N}}$ of the eigenvalues and eigenfunctions of the corresponding Sturm-Liouville equation with Dirichlet boundary conditions to represent $u^{f}$ as follows:

$$
u^{f}(t, x)=\sum_{i=1}^{\infty} u_{i}^{f}(t) \phi_{i}(x)
$$

where the Fourier coefficients $u_{i}^{f}(t):=\int_{0}^{1} u^{f}(t, x) \phi_{i}(x) d x$ is completely characterized by the spectral data $\left(\phi_{n}^{\prime}(0), \lambda_{n}\right)_{n \in \mathbb{N}}$, i.e. $u_{i}^{f}(t)=\left(\phi_{n}\right)^{\prime}(0) \int_{0}^{1} f(s) \frac{\sin \left(\sqrt{\lambda_{i}}(t-s)\right)}{\sqrt{\lambda_{i}}} d s$, since it is the solution of the Cauchy problem ${ }^{4}$

$$
\left\{\begin{array}{l}
\frac{\partial^{2} u^{f}}{\partial t^{2}}-\lambda_{i} u^{f}=\left(\phi_{n}\right)^{\prime}(0) f(t), \text { in }(0, T), \\
u_{i}^{f}(0)=\frac{d}{d t} u_{i}^{f}(t)=0, t \in(0, T) .
\end{array}\right.
$$

3. Boundary controllability. There are two types of boundary controllability. First, the exact boundary controllability for the problem (24) is to find, for every fixed $t \in(0, T)$, for every $z(x)$ in $L^{2}\left(\Gamma_{t}\right)$ a function (i.e. a control) $f \in L^{2}(0, T)$ such that $u^{f}(t, x)=z(x)$. Second, we have the approximate boundary controllability where we replace the equality $u^{f}(T, x)=z(x)$ by an approximation, see [5] for instance. The second property is enough for our purpose.

Based on these three arguments, we prove the following theorem which characterizes fully the eigenfunctions $\phi_{n}$ in $\Gamma_{t}$, for every $t \leq 1$ using only the Gelfand spectral data.

Theorem 3. Let $V_{j}, j=1,2$ be two potentials such that the corresponding Gelfand spectral data $\left(\lambda_{i}^{j},\left|\left(\phi_{i}^{j}\right)^{\prime}(0)\right|\right)_{i \in \mathbb{N}}, j=1,2$, are equal. Then, we have

\footnotetext{
${ }^{3}$ For the general form Sturm-Liouville model (1), the speed of propagation is $\int_{0}^{x} \sqrt{\frac{\rho}{p}}(t) d t$ hence
} $\Gamma_{t}=\left\{x \in(0,1), \int_{0}^{x} \sqrt{\frac{\rho}{p}}(t) d t<t\right\}$.

${ }^{4}$ Replace $\sqrt{\lambda_{i}}$ by $\sqrt{\left|\lambda_{i}\right|}$ for possible negative eigenvalues $\lambda_{i}$ or $\frac{\sin \left(\sqrt{\lambda_{i}}(t-s)\right)}{\sqrt{\lambda_{i}}}$ by $t-s$ if $\lambda_{i}=0$. 


$$
\int_{0}^{t}\left(\phi_{i}^{1}\right)^{2}(x) d x=\int_{0}^{t}\left(\phi_{i}^{2}\right)^{2}(x) d x, \forall i \in \mathbb{N}, \forall t \in(0, T) .
$$

From (27), we have $\left(\phi_{i}^{1}\right)^{2}(x)=\left(\phi_{i}^{2}\right)^{2}(x)$, for $x \in[0, T]$ (or for $x \in[0,1]$ if $T \geq 1$ ) and $i \in \mathbb{N}$. Taking $i=1$, we have $V_{1}=\frac{\left(\phi_{1}^{1}\right)^{\prime \prime}-\lambda_{1} \phi_{1}^{1}}{\phi_{1}^{1}}=\frac{\left(\phi_{1}^{2}\right)^{\prime \prime}-\lambda_{1} \phi_{1}^{2}}{\phi_{1}^{2}}=V_{2}$ in $(0,1)$ knowing that the eigenfunction $\phi_{1}^{j}$ never vanish in $(0,1)$.

The proof of Theorem 3 goes as follows. Let $\left(f_{k}\right)_{k \in \mathbb{N}}$ be a dense set in $H_{0}^{1}(0,1)$. From the well posedness of the problem (24) and the approximate boundary controllability, we deduce that finite combinations of the functions $u^{f_{k}}(t, x)$ is dense in $L^{2}(0, t)$. From the second argument we know that the Fourier coefficients of $u^{f_{k}}$ can be reconstructed from the Gelfand spectral data. By a Gram-Schmidt orthonormalization procedure, we can find an orthogonal basis of $L^{2}(0, t)$ given by combinations of $u^{f_{k}}$, i.e. $v_{s}:=\sum_{1}^{n(s)} d_{s} u^{f_{i}}$. By linearity, we have $v_{s}=u^{g_{s}}$ where $g_{s}:=\sum_{1}^{n(s)} d_{s} f_{i}$. Now, we write $\phi_{j}=\sum_{k=1}^{\infty}\left[\int_{0}^{t} \phi_{j}(x) v_{k}(t, x) d x\right] v_{k}(t, x)$ in $(0, t)$ and hence

$$
\int_{0}^{t} \phi_{i}(x) \phi_{j}(x) d x=\sum_{k=1}^{\infty} \int_{0}^{t} \phi_{j}(x) v_{k}(t, x) d x \int_{0}^{t} v_{k}(t, x) \phi_{i}(x) d x .
$$

Again from the second argument above, we know that

$$
\int_{0}^{t} \phi_{j}(x) v_{k}(t, x) d x=\left(\phi_{j}\right)^{\prime}(0) \int_{0}^{t} g_{k} \frac{\sin \sqrt{\lambda_{j}}(t-s)}{\sqrt{\lambda_{j}}} d s .
$$

Taking $i=j$ in (28) and using (29), we see that the Gelfand spectral data completely characterize the quantities $\int_{0}^{t}\left(\phi_{i}(x)\right)^{2} d x, i \in \mathbb{N}$. This ends the proof of Theorem 3 .

\section{References}

1. M. I. Belishev (2007) Recent progress in the boundary control method. Inverse Problems 23 (5), $\mathrm{R} 1-\mathrm{R} 67$.

2. G. Borg (1946) Eine Umkerung der SturmLiouville Eigenwertarfgabe. Acta Math. 78, 1-96.

3. O. H. Hald and J. R. McLaughlin (1989) Solutions of inverse nodal problems. Inverse Problems 5 (3), 307-347. 
4. I. M. Gel'fand and B. M. Levitan (1951) On the determination of a differential equation from its special function, Izv. Akad. Nauk SSR. Ser. Mat. 15, 309-360 (Russian); English transl. in Amer. Math. Soc. Transl. Ser. 2 (1) (1955), 253-304.

5. A. Katchalov; Y. Kurylev and M. Lassas (2001) Inverse boundary spectral problems. Chapman/Hall/CRC Monographs and Surveys in Pure and Applied Mathematics, 123. Chapman/Hall/CRC, Boca Raton, FL, xx+290 pp.

6. A. Kirsch (1996) An introduction to the mathematical theory of inverse problems. Applied Mathematical Sciences, 120. Springer-Verlag, New York, x+282 pp.

7. N. Levinson (1949) The inverse Sturm-Liouville problem Math. Tidsskr. B 25 25-30.

8. B. M. Levitan (1987) Inverse Strum-Liouville problems. VNU Science Press, Ultrecht.

9. J. R. McLaughlin (1986) Analytical methods for recovering coefficients in differential equations from spectral data. SIAM Rev. 28 (1), 53-72.

10. J. Poschel and E. Trubowitz (1987) Inverse spectral theory. Pure and Applied Mathematics, 130. Academic Press, Inc., Boston, MA, x+192 pp.

11. A. Ramm (2004) Inverse Problems. Mathematical and Analytical Techniques with Applications to Engineering, Springer.

12. W. Rundell and P. Sacks (1992) Reconstruction techniques for classical inverse Sturm-Liouville problems. Math. Comp. 58, no. 197, 161-183.

13. M. Sini (2003) Some uniqueness results of discontinuous coefficients for the one-dimensional inverse spectral problem. Inverse Problems 19 (4), 871-894. 